\title{
Study of Numerical Simulation during ECAP Processing of Can Based on Smooth Particle Hydrodynamics
}

\author{
Xiaofeng Niu $\mathbb{D}^{1},{ }^{1}$ Chenchen Wang, ${ }^{1}$ K. C. Chan ${ }^{(D)},{ }^{2}$ Han Wang, ${ }^{3}$ and Shidong Feng ${ }^{2,4}$ \\ ${ }^{1}$ College of Materials Science and Engineering, Taiyuan University of Technology, Taiyuan 030024, China \\ ${ }^{2}$ Advanced Manufacturing Technology Research Centre, Department of Industrial and Systems Engineering, \\ The Hong Kong Polytechnic University, 999077, Hong Kong \\ ${ }^{3}$ Hebei Institute of Metrological Supervision \& Measurement/Hebei Institute of Metrology, Shijiazhuang, 050051, China \\ ${ }^{4}$ State Key Laboratory of Metastable Materials Science and Technology, Yanshan University, Qinhuangdao 066004, China
}

Correspondence should be addressed to Xiaofeng Niu; niuxiaofeng@tyut.edu.cn and K. C. Chan; kc.chan@polyu.edu.hk

Received 9 February 2019; Revised 14 April 2019; Accepted 17 April 2019; Published 22 May 2019

Academic Editor: Dimitri Volchenkov

Copyright (C) 2019 Xiaofeng Niu et al. This is an open access article distributed under the Creative Commons Attribution License, which permits unrestricted use, distribution, and reproduction in any medium, provided the original work is properly cited.

ECAP (Equal Channel Angular Pressing) is a well-known technique by which a specimen is pressed into an ECAP die to improve the mechanical properties by the nearly pure shear during the deformation process. In the ECAP processing of can, the specimen is canned with a protection material layer to avoid the cracking during deformation. At present, most simulation studies of ECAP are conducted based on the finite element method, in which large deformation can cause serious mesh distortion, resulting in a decrease of the simulation accuracy. In this study, based on SPH (Smooth Particle Hydrodynamics), we utilize the invalid particles and crack treatment techniques, building an ECAP mathematical model incorporating damage prediction, in order to simulate crack initiation and dynamic extension in the ECAP process. In simulation of pure magnesium during ECAP at room temperature using industrial pure iron as the canned material, the simulation results based on SPH method show that the plastic deformation of the pure magnesium specimen is homogeneous in both the vertical direction and the extrusion direction. The average equivalent strain value of the specimen in the major deformation area is 1.31 , which is similar to the finite element simulation result in which the average equivalent strain value of the major deformation area is 1.24 . From the damage perspective, the maximum damage values of the inside specimen obtained by the SPH method and the finite element method are both less than 0.16 , with both values being far lower than the critical fracture accumulated damage value. The test results well match the simulation results.

\section{Introduction}

In order to prevent the magnesium and magnesium alloy from cracking during the ECAP process, the deformation temperature is maintained higher than $200^{\circ} \mathrm{C}[1,2]$. The focus is on how to improve the plastic deformation performance when reducing the pressing temperature of the magnesium and magnesium alloy $[3,4]$. As an improvement of the ECAP technology, canned ECAP technology is applied in the preparation of ultrafine grain materials and is verified as being highly effective. In the ECAP processing of can [5], the specimen is canned with a protection material layer to avoid the cracking of the specimen during the ECAP deformation [6].

At present, most simulation studies of ECAP are conducted based on the finite element method; e.g., Yoon [7] studied the plastic deformation behavior of materials in the back pressure ECAP process by using the finite element method and, respectively, analyzed the influences of friction, strain hardening, and the outer corner on the deformation homogeneity of the specimen after processing by ECAP. Djavanroodi [8] analyzed the influences of different technological parameters on the ECAP process by using the finite element method and demonstrated that the deformation homogeneity can be improved by increasing the friction coefficient or applying a back pressure. Before using the damage prediction model [9] to simulate the ECAP process based on the finite element method, it is required to undertake mesh refinement within the areas where the specimen is likely to initiate cracks, by analyzing the distribution of damage factors, and then distribute crack origins across such areas filled with refined meshes. It is inevitable to decrease the 
accuracy of computation due to the distribution of the crack origins and being unable to accurately predict the initiation positions or dynamic extension of the cracks. Additionally, the large deformation of material during the process will lead to serious distortion of the meshes, resulting in decreased accuracy of simulation.

The SPH is a mesh-free numerical simulation method [10-14], which has attracted great attention in recent years. Based on the Lagrangian description, this method does not need the mesh to be divided and thereby can eliminate the decreased solution accuracy, divergent numerical values, and other problems caused by the generation, regeneration, entanglement, or distortion of meshes. It is applied in computations which cannot be solved by using the traditional finite element method. SPH is not restricted by the meshes and uses discrete particles for computing, which can eliminate the decreased computation accuracy caused by the mesh distortion in the mesh based methods. Pan [15] conducted a simulation study on the friction stir welding of magnesium alloy by using SPH and introduced SPH into the material flow field successfully. Cleary [16] studied the metal forging process by using SPH and demonstrated the feasibility of using SPH for simulating the material flow and deformation in the forging process.

This paper, by using the SPH method and taking into account crack treatment techniques, builds an ECAP mathematical model incorporating damage prediction, to simulate crack initiation and dynamic extension in the ECAP process. By taking pure magnesium as an example and leveraging the invalid particles, we analyze the deformation and damage process of pure magnesium during the room temperature ECAP process from the macroscopic perspective. The room temperature canned ECAP process is studied and analyzed, with the selection of the canned material, and verifies the validity and effectiveness of computation model.

\section{Mathematical Model}

2.1. Basic Theories of SPH. SPH is a numerical method based on integral interpolation. The integral interpolation of an arbitrary field function $A(r)$ can be expressed as follows [17]:

$$
A\left(\boldsymbol{r}_{i}\right)=\int_{\Omega} A\left(\boldsymbol{r}^{\prime}\right) W\left(\boldsymbol{r}_{i}-\boldsymbol{r}^{\prime}, h\right) d \boldsymbol{r}^{\prime}
$$

wherein $A$ represents any function of any point $r_{i} ; \Omega$ denotes the integral domain associated with the space of $r_{i}$; $r^{\prime}$ represents the position vector; $d r^{\prime}$ is the volume element at the point $r^{\prime} ; W\left(r_{i}-r^{\prime}, h\right)$ is called smooth function or kernel function; and $h$ indicates the smooth length, which defines the range of support domain and thereby determines the computation efficiency and accuracy during the SPH solving process.

The particle approximation of field function at particle $i$ can be expressed as

$$
A\left(\boldsymbol{r}_{i}\right)=\sum_{j=1}^{N} \frac{m_{j}}{\rho_{j}} A\left(\boldsymbol{r}_{j}\right) W_{i j}
$$

wherein $\sum_{j=1}^{N}$ denotes the summation of influences of particle $j$ on particle $i$ within the support domain; $m_{j}$ is the mass of particle $j ; \rho_{j}$ is the density of particle $j$; and $W_{i j}=$ $W\left(r_{i}-r^{\prime}, h\right)$ represents the smoothing function. This paper adopts the cubic spline function.

2.2. ECAP Mathematical Model Incorporating Damage Prediction. Based on SPH and by utilizing the invalid particles and crack treatment techniques, this paper builds an ECAP mathematical model incorporating damage prediction to simulate the crack initiation and dynamic extension in the ECAP process. When the SPH method is used for describing the ECAP process of a material, it is required to introduce the constitutive model of metal material for computing the effect of stress and strain condition on the strength of the material. Meanwhile, the metal material will show weak compressibility during the test, for which a state equation is needed to describe the real deformation process of the metal material subject to the pressure of upper die during the ECAP process. In order to facilitate the computation, presume that the contact between two parts of the specimen, the can and die, is smooth without friction. The boundary treatment is performed based on the penalty function method. The density, velocity, and position of the particles are updated by the Leap-Frog method [18].

The total stress tensor $\sigma^{\alpha \beta}$ is composed of pressure $P$ and deviator stress tensor $\tau^{\alpha \beta}$.

$$
\sigma^{\alpha \beta}=-P \delta^{\alpha \beta}+\tau^{\alpha \beta}
$$

In this equation, $\delta$ denotes the Dirac function [19] and $P$ is the pressure. The SPH method is compressible with the pressure calculated from the particle density using an equation of state:

$$
P=c_{0}^{2}\left(\rho-\rho_{0}\right)
$$

where $c_{0}$ denotes the sound velocity [19]; $\rho_{0}$ is the initial density of the particle; and $\rho$ is the density of particle at the present moment, with $\mathrm{d} \rho_{i} / \mathrm{d} t$ calculated as follows:

$$
\frac{\mathrm{d} \rho_{i}}{\mathrm{~d} t}=\sum_{j=1}^{N} m_{j} v_{i j}^{\beta} \frac{\partial W_{i j}}{\partial x_{i}^{\beta}}
$$

where $d \rho_{i} / d t$ represents the density variation rate of particle $i ; \sum_{j=1}^{N}$ denotes the summation of the influences of particle $j$ on particle $i$ within the support domain; $m_{j}$ is the mass of the particle $j ; v_{i j}$ is the velocity difference between particle $i$ and particle $j$; the Greek letters $\alpha, \beta, \gamma$ indicate the coordinate directions, using the index method to indicate the superposition of equation $[18,19]$; and $W_{i j}$ is a smoothing function.

According to solid mechanics, the stress is expressed as a function of strain and strain rate, and the Jaumann rate is used to express the relation between the deviator stress tensor and strain rate as

$$
\dot{\tau}^{\alpha \beta}=2 G\left(\varepsilon^{\alpha \beta}-\frac{1}{3} \delta^{\alpha \beta} \varepsilon^{\gamma \gamma}\right)+\tau^{\alpha \gamma} R^{\beta \gamma}+\tau^{\gamma \beta} R^{\alpha \gamma}
$$


where $G$ denotes the shear modulus; $\dot{\tau}^{\alpha \beta}$ is the tensor of deviator stress rate; $\varepsilon^{\alpha \beta}$ is the tensor of strain rate; and $R^{\alpha \beta}$ is the tensor of torsion rate. The SPH expression of strain rate tensor and torsion rate tensor is shown as follows.

$$
\begin{aligned}
\varepsilon_{\mathrm{i}}^{\alpha \beta} & =\frac{1}{2} \sum_{j=1}^{N}\left(\frac{m_{j}}{\rho_{j}} v_{i j}^{\alpha} \frac{\partial W_{i j}}{\partial x_{i}^{\beta}}+\frac{m_{j}}{\rho_{j}} v_{i j}^{\beta} \frac{\partial W_{i j}}{\partial x_{i}^{\partial}}\right) \\
R_{\mathrm{i}}^{\alpha \beta} & =\frac{1}{2} \sum_{j=1}^{N}\left(\frac{m_{j}}{\rho_{j}} v_{i j}^{\alpha} \frac{\partial W_{i j}}{\partial x_{i}^{\beta}}-\frac{m_{j}}{\rho_{j}} v_{i j}^{\beta} \frac{\partial W_{i j}}{\partial x_{i}^{\partial}}\right)
\end{aligned}
$$

Since the yield strengths of magnesium and magnesium alloy materials are greatly influenced by the stress, strain rate and temperature and damage are also varying greatly with the deformation conditions, so that the constitutive model proposed by Johnson and Cook, based on the accumulated damage values, is adopted to express the yield strength of the metal as follows:

$$
\begin{aligned}
Y= & \left(1-D_{s}\right) \cdot\left[A+B\left(1-D_{s}\right) \varepsilon_{p}^{n}\right] \\
& \cdot\left[1+C \ln \left(\left(1-D_{s}\right) \frac{\dot{\varepsilon}_{p}}{\dot{\varepsilon}_{0}}\right)\right]\left[1-\left(T^{*}\right)^{m}\right]
\end{aligned}
$$

where $Y$ denotes the yield strength; $\varepsilon_{p}$ is the equivalent plastic strain obtained from the integration of equivalent plastic strain rate $\dot{\varepsilon}_{p} ; \dot{\varepsilon}_{0}$ is a reference strain rate, which is set as $1 \mathrm{~m} \cdot \mathrm{s}^{-1}$ herein; $A$ denotes the initial yield stress; $B$ and $n$ are the strain hardening parameters of metal material; and $C$ is the coefficient for the strain rate effect of metal material. The equivalent plastic strain rate $\dot{\varepsilon}_{p}$ is expressed as follows.

$$
\dot{\varepsilon}_{p}=\sqrt{\frac{2}{3}\left(\varepsilon^{x x} \times \varepsilon^{x x}+\varepsilon^{y y} \times \varepsilon^{y y}+\varepsilon^{z z} \times \varepsilon^{z z}+2 \varepsilon^{x y} \times \varepsilon^{x y}+2 \varepsilon^{x z} \times \varepsilon^{x z}+2 \varepsilon^{y z} \times \varepsilon^{y z}\right)}
$$

$m$ is a coefficient for describing the thermal softening of the metal, and $T^{*}$ denotes the nondimensional temperature:

$$
T^{*}= \begin{cases}\frac{T-T_{r}}{T_{m}-T_{r}}, & T<T_{m} \\ 0, & T \geq T_{m}\end{cases}
$$

where $T$ is the current temperature of the metal and a fixed value is set in order to simplify the mathematical model; $T_{m}$ is the melting point of the material; and $T_{r}$ denotes the room temperature, set as $20^{\circ} \mathrm{C}$ herein.

In (9), $D_{s}$ is the accumulated damage value. When the accumulated damage value $D_{s}$ exceeds the critical fracture accumulated damage value $D_{s c}$, the material will fracture. According to testing, the critical fracture accumulated damage value $D_{s c}$ of the material is less than or equal to 1.0.

In order to exactly describe the process from the crack initiation to extension of the material in the ECAP process, we adopt the damage evaluation model corrected by Børvik [20, 21]. This model utilizes the accumulated equivalent plastic strain to judge if the damage starts to evolve, and expresses the accumulated damage value change rate $\dot{D}_{s}$ as

$$
\dot{D}_{s}= \begin{cases}\frac{D_{s c}}{\varepsilon_{f}-\varepsilon_{d}} \dot{\varepsilon}_{p}, & \varepsilon_{p} \geq \varepsilon_{d} \\ 0, & \varepsilon_{p}<\varepsilon_{d}\end{cases}
$$

where $\varepsilon_{d}$ is the accumulated value of equivalent strain when the material damage begins to evolve. The accumulated equivalent strain of pure magnesium at the beginning of evolution is set herein as $1 \times 10^{-6}$, and $\varepsilon_{f}$ denotes the critical fracture equivalent strain, expressed as follows:

$$
\begin{aligned}
\varepsilon_{f}= & {\left[D_{1}+D_{2} \exp \left(D_{3} \eta\right)\right]\left[1+D_{4} \ln \left(\frac{\dot{\varepsilon}_{p}}{\dot{\varepsilon}_{0}}\right)\right] } \\
& \cdot\left[1+D_{5} T^{*}\right]
\end{aligned}
$$

where the damage parameters $D_{1}, D_{2}$, and $D_{3}$ describe the influence of material's stress hardening on the crack extension; $D_{4}$ describes the material's strain rate effect; $D_{5}$ describes the material's thermal softening; and $\eta$ is the stress triaxiality, which is obtained from the ratio of pressure $P$ to the equivalent stress $\sigma_{e q}$. The equivalent stress $\sigma_{e q}$ can be calculated by the following.

$$
\sigma_{e q}=\sqrt{\frac{3}{2}\left(\tau^{x x} \times \tau^{x x}+\tau^{y y} \times \tau^{y y}+\tau^{z z} \times \tau^{z z}+2 \times \tau^{x y} \times \tau^{x y}+2 \tau^{x z} \times \tau^{x z}+2 \tau^{y z} \times \tau^{y z}\right)}
$$

Assume that the material to be deformed during the ECAP process is an ideal elastic-plastic material, in which the equivalent stress $\sigma_{e q}$ satisfies the following.

$$
\sigma_{e q}>Y
$$

At this moment, the particle $i$ enters into the material's plastic yield state. The deviator stress tensor is corrected by using the equivalent stress of particle $i$ and the yield strength of the material as follows.

$$
\tau^{\alpha \beta}=\tau^{\alpha \beta} \frac{Y}{\sigma_{e q}}
$$

Due to the severe deformation of material during the ECAP process, the computation result is very likely to 
show nonphysical oscillation, and therefore it is necessary to introduce kinetic energy-thermal energy conversion, namely, the artificial viscosity. Liu [19] introduces artificial viscosity $\Pi_{i j}$, which is widely applied at present and is used herein. Additionally, to eliminate the tensile instability, the artificial stress term $\widetilde{R}_{i j}^{\alpha \beta} \widetilde{f}_{i j}^{n}$ is introduced $[18,22,23]$. The artificial viscosity $\Pi_{i j}$ and artificial stress term $\widetilde{R}_{i j}^{\alpha \beta} \widetilde{f}_{i j}^{n}$ are, respectively, added to the SPH momentum equation to obtain the final SPH momentum equation as follows:

$$
\frac{\mathrm{d} v_{i}^{\alpha}}{\mathrm{d} t}=-\sum_{j=1}^{N} m_{j}\left(\frac{\sigma_{i}^{\alpha \beta}}{\rho_{i}^{2}}+\frac{\sigma_{j}^{\alpha \beta}}{\rho_{j}^{2}}+\Pi_{i j}+\widetilde{R}_{i j}^{\alpha \beta} \widetilde{f}_{i j}^{n}\right) \frac{\partial W_{i j}}{\partial x_{i}^{\beta}}
$$

where $\mathrm{d} v_{i}^{\alpha} / \mathrm{d} t$ is the acceleration of particle $i ; \widetilde{f}_{i j}^{n}$ in the artificial stress term is a function in direct proportion to the particle spacing; and $\widetilde{R}_{i j}^{\alpha \beta}$ is a function of stress and density.

Similarly, the expression of energy calculation is as follows [19].

$$
\frac{\mathrm{d} e_{i}}{\mathrm{~d} t}=\frac{1}{2} \sum_{j=1}^{\mathrm{N}} m_{j}\left(\frac{P_{i}}{\rho_{i}^{2}}+\frac{P_{j}}{\rho_{j}^{2}}+\Pi_{i j}\right) v_{i j}^{\beta} \frac{\partial W_{i j}}{\partial x_{i}^{\beta}}+\frac{1}{\rho_{i}} \tau_{i}^{\alpha \beta} \varepsilon_{i}^{\alpha \beta}
$$

In order to intuitively reflect the dynamic crack extension of the metal, inspired by the idea of deleting the mesh cells for simulating the crack extension in the finite element method, we set the SPH particles with the accumulated damage value $D_{s}$ reaching the critical fracture accumulated damage value $D_{s c}$ as invalid particles. Computing the interaction between the invalid particles and other particles is stopped, so as to realize the simulation of the crack initiation and dynamic extension during the ECAP process. Meanwhile, in order to avoid the overlap between the invalid particles inconsistency with the actual conditions and other particles, we introduce the boundary repulsive force model proposed by Monaghan [24] to apply a Lennard-Jones pair-potential repulsive force $f_{i j}^{N}$ onto the particle just at the moment when it becomes invalid. The repulsive force $f_{i j}^{N}$ is expressed as

$$
\begin{aligned}
& f_{i j}^{N} \\
& = \begin{cases}\widetilde{D} \cdot\left(\left(\frac{r_{0}}{\left|x_{i j}\right|}\right)^{n_{1}}-\left(\frac{r_{0}}{\left|x_{i j}\right|}\right)^{n_{2}}\right) \cdot \frac{x_{i j}}{\left|x_{i j}\right|^{2}} & \left(\frac{r_{0}}{\left|x_{i j}\right|}\right)<1 \\
0 & \left(\frac{r_{0}}{\left|x_{i j}\right|}\right) \geq 1\end{cases}
\end{aligned}
$$

where the particle $i$ is an internal particle; the particle $j$ is an invalid particle; $\widetilde{D}$ equals the squared value of the maximum velocity of the invalid particle; $\left|\boldsymbol{x}_{\boldsymbol{i}}\right|$ denotes the distance between the particle and the invalid particle; $x$ is a position vector, with $\boldsymbol{x}_{\boldsymbol{i}}=\boldsymbol{x}_{\boldsymbol{i}}-\boldsymbol{x}_{\boldsymbol{j}}$; typically, we set the parameters $n_{1}=12$ and $n_{2}=4$; and $r_{0}$ denotes the cutoff radius of the invalid particle repulsive force, which is set as 2 times the particle diameter herein. Since the invalid particle does not participate in the computation within the whole problem domain, the computation result of the whole problem domain

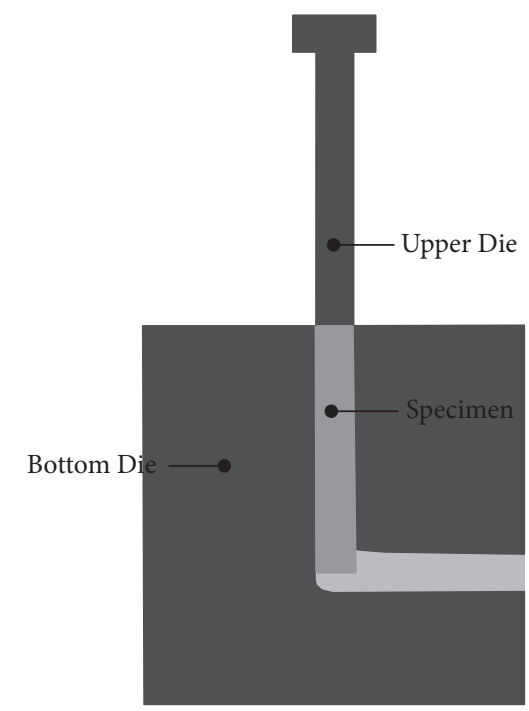

FIGURE 1: Structural diagram of ECAP dies.

will not be influenced by the nonconservation of the invalid particle momentum equation resulting from the repulsive force calculated by using the Lennar-Jones model.

\section{Simulation Results and Analysis}

Based on SPH and by utilizing the invalid particles and crack treatment techniques, an ECAP mathematical model incorporating damage prediction to simulate the crack initiation and dynamic extension in the ECAP process is built. By taking pure magnesium as an example, we first conducted a numerical simulation of the room temperature ECAP process without can to verify the feasibility and validity of the mathematical model. A numerical simulation of room temperature ECAP process with can is conducted to demonstrate that the types of external can materials will directly influence the plastic deformation of internal specimen. The combined specimen canned with 2024 aluminum alloy generates lamellar cracks, while the combined specimen canned with industrial pure iron maintains the integrity.

This study adopts the ECAP dies as shown in Figure 1. In order to further verify the simulation results, our research group conducted some controlled tests $[25,26]$. The equipment of ECAP (see Figure 2) includes ECAP dies and a computer-controlled electronic universal testing machine (CMT5205). Figure 3 shows the internal structure of the bottom die. Both channels of the bottom die are square channels with a cross section of $10 \mathrm{~mm} \times 10 \mathrm{~mm}$. The crossing angle is $90^{\circ}$, and there is a $3^{\circ}$ deflection angle structure above the horizontal channel. The die is made of tool steel $(\mathrm{Cr} 12 \mathrm{MoV})$ to prevent any deformation during the process. In the simulation calculation, the setting of the extrusion velocity is expressed by giving the velocity value of the moving boundary.

3.1. Numerical Simulation of Room Temperature ECAP Process of Pure Magnesium without Can. The specimen, under the pressure of upper die, enters into the horizontal channel 




FIGURE 2: Equipment of ECAP.

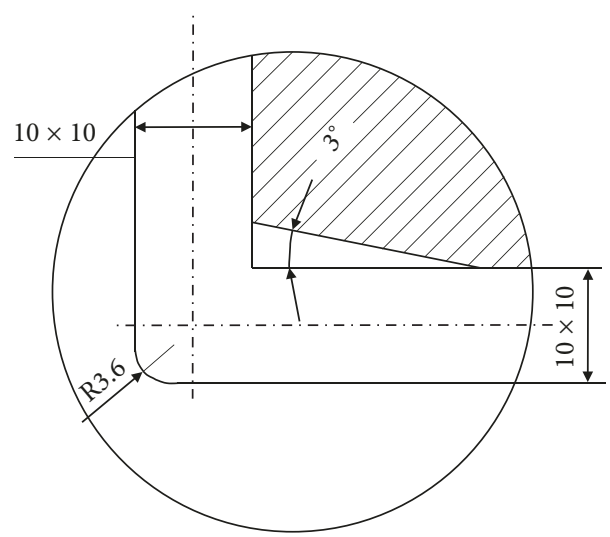

FIGURE 3: Internal structure of bottom die.

through the vertical channel of the bottom die. The cross sections of these two channels have the same geometrical shape. When passing the intersection between the two channels, the specimen suffers approximately ideal simple shear to generate large shear deformation to, thereby, produce the ultrafine grain material. The dimension of the specimen to be deformed is $10 \mathrm{~mm} \times 10 \mathrm{~mm} \times 50 \mathrm{~mm}$. To facilitate the computation, it is assumed that there is no friction between the die and material.

Discretely, the material to be deformed is split into a series of particles, as shown in Figure 4. The number of particles is 20,000 . The dark blue area represents the deformed material. The upper die is simplified into the compression movable boundary composed of a single layer of red particles as shown, while the bottom die is simplified into a fixed wall boundary composed of a single layer of black particles. The penalty function method is adopted to calculate the repulsive force. The total number of particles in two boundaries is 828 . The initial spacing between particles is $0.25 \mathrm{~mm}$. The smoothing length is set as 1.5 times the initial particle spacing. In order to obtain the parameters for the Johnson-Cook damage



FIGURE 4: ECAP initial model in SPH method.

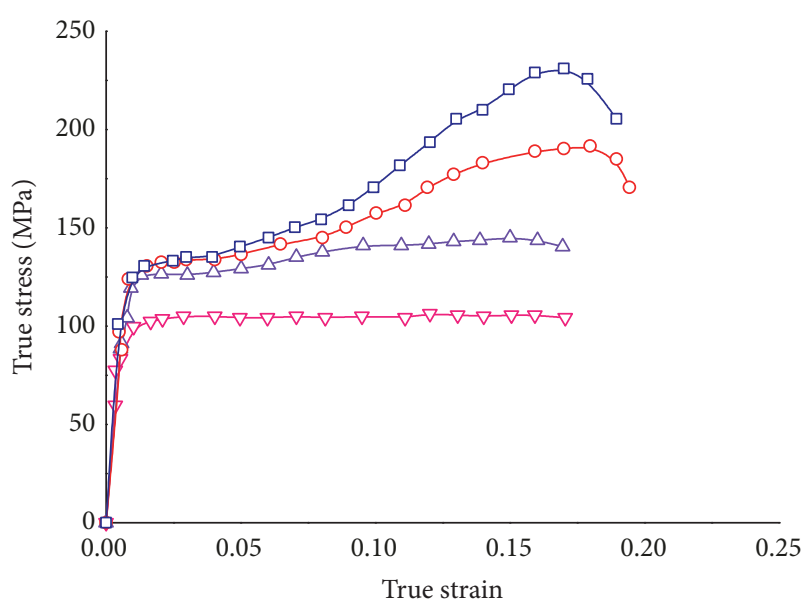

$$
\begin{gathered}
\left(\mathrm{s}^{-1}\right) \\
-\nabla-10^{-5} \\
-\triangle-10^{-4} \\
-\circ-10^{-3} \\
-\square-10^{-2}
\end{gathered}
$$

Figure 5: True stress-strain curve of as-extruded pure magnesium compression at room temperature.

constitutive model of pure magnesium at room temperature, a unidirectional compression test was conducted on the pure magnesium specimen. Figure 5 shows the true stress-strain curve of as-extruded pure magnesium.

Based on the above test data, the parameters for JohnsonCook damage model of pure magnesium were obtained as listed in Table 1, using the method for estimating the parameters of material constitutive model described in [27, 28]. Conducting the deformation of pure magnesium material is discussed herein at room temperature, without considering the influence of temperature on the critical fracture strain of pure magnesium; $D_{5}$ is set as 0.0 .

Adopting the program provided herein for the SPH based model, incorporating damage prediction to solve the 


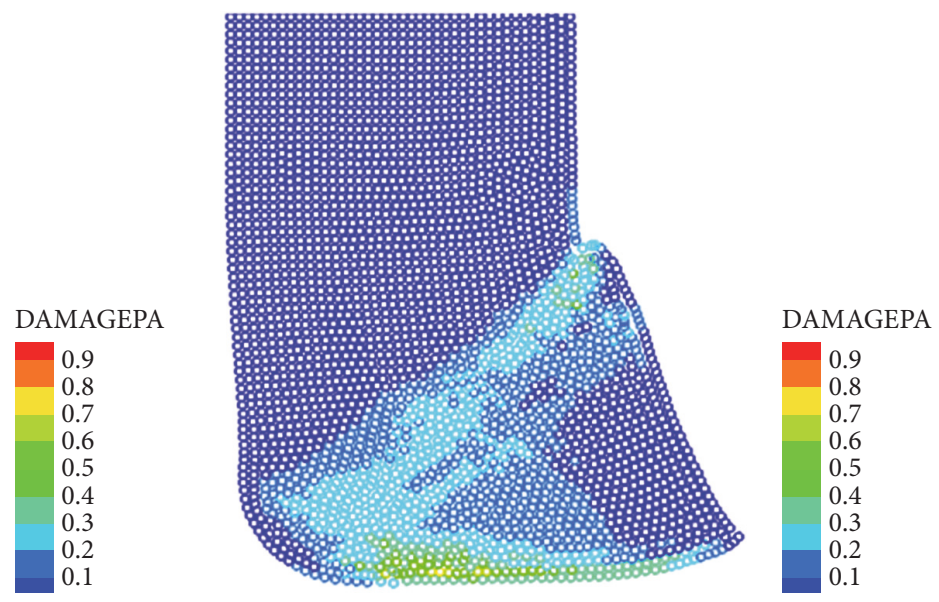

(a)

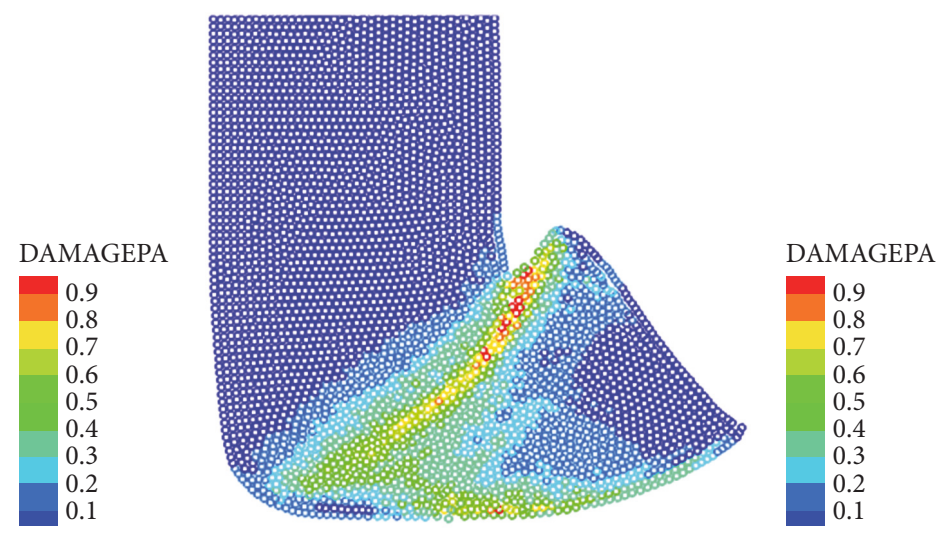

(c)

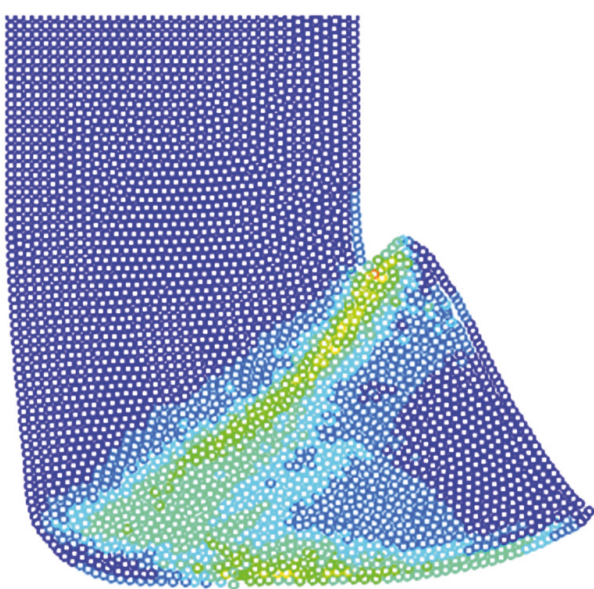

(b)

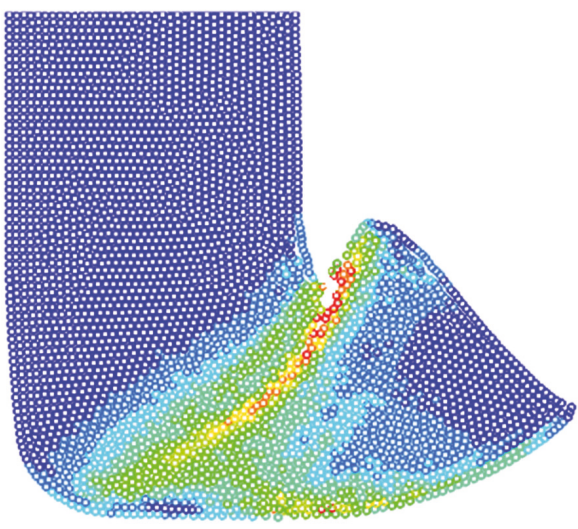

(d)

FIGURE 6: Simulation results of accumulated damage value distribution.

TABLE 1: Parameters for constitutive model of pure magnesium.

\begin{tabular}{lccccccc}
\hline Material & $\rho\left(\mathrm{kg} \cdot \mathrm{m}^{-3}\right)$ & $v$ & $A(\mathrm{MPa})$ & $B(\mathrm{MPa})$ & $\mathrm{C}$ & $m$ & $n$ \\
\hline pure magnesium & 1780 & 0.35 & 45 & 110 & 0.059 & 1.49 & $D_{3}$ \\
\hline$T_{r}(\mathrm{~K})$ & $T_{m}(\mathrm{~K})$ & $C_{v}$ & $D_{1}$ & $D_{2}$ & 0.16 \\
\hline 293 & 933 & 1050 & 0.0205 & -1.782 & -0.421 & 0.012 & 0.0 \\
\hline
\end{tabular}

extrusion velocity as $1 \mathrm{~mm} \cdot \mathrm{s}^{-1}$ pure magnesium ECAP deformation, gives the computation results of the accumulated damage value distribution as shown in Figure 6.

Figure 6 shows the accumulated damage value distribution of pure magnesium during room temperature ECAP deformation at an extrusion velocity of $1 \mathrm{~mm} \cdot \mathrm{s}^{-1}$. As seen in Figure 6(a), along with the movement of the movable boundary, the specimen enters into the plastic deformation area and begins the evolution process. A large accumulated damage value occurs at the bottom, at first, but does not reach the critical fracture accumulated damage value. Along with the extrusion, the accumulated damage value of the particles within the area close to the internal corner clearly increases, as shown in Figure 6(c), and a large damage value occurs within the area within the vicinity of the shear plane. The particle is converted into an invalid particle when the accumulated damage value reaches the critical fracture accumulated damage value, and a crack is initiated on the surface of the specimen close to the internal corner of die and then extends along the shear plane, as shown in Figure 6(d).

Figure 7 shows the equivalent strain distribution of pure magnesium deformation during room temperature ECAP at an extrusion velocity of $1 \mathrm{~mm} \cdot \mathrm{s}^{-1}$. As shown in Figure 7(b), the high strain concentration area also occurs on the shear plane of the pure magnesium specimen and forms a visible plastic deformation band on the shear plane. As shown in Figure $7(\mathrm{c})$, along with the continuous compression of the upper die, the equivalent strain value of the specimen increases sharply within the area close to the internal corner of the channel, resulting in the high strain concentration along the shear plane. The equivalent strain value of the particle on the specimen along the shear plane shows a tendency of decreasing gradually from the internal corner to the outer corner. Along with the increase of the equivalent 


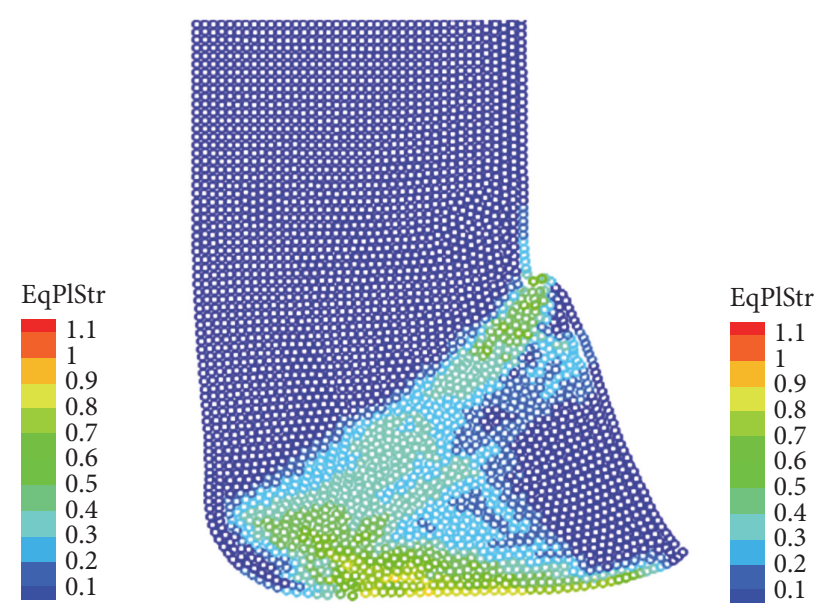

(a)
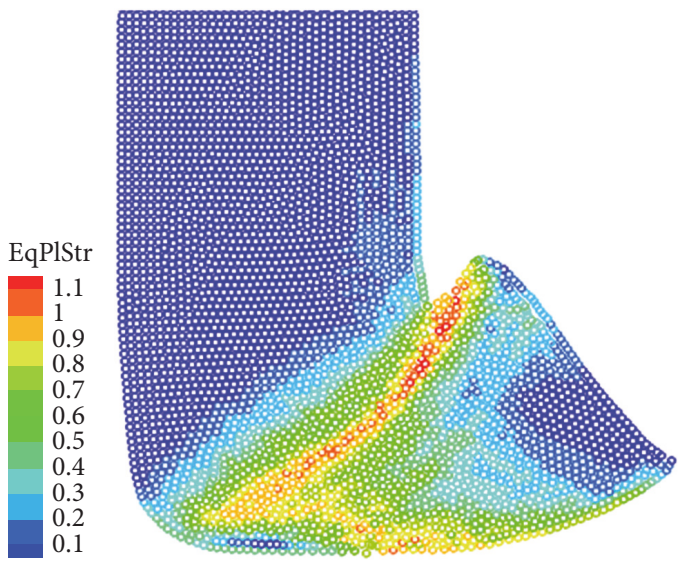

(c)

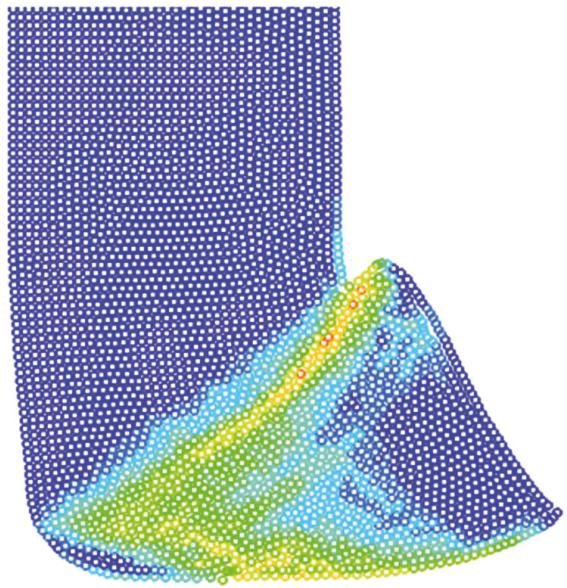

(b)

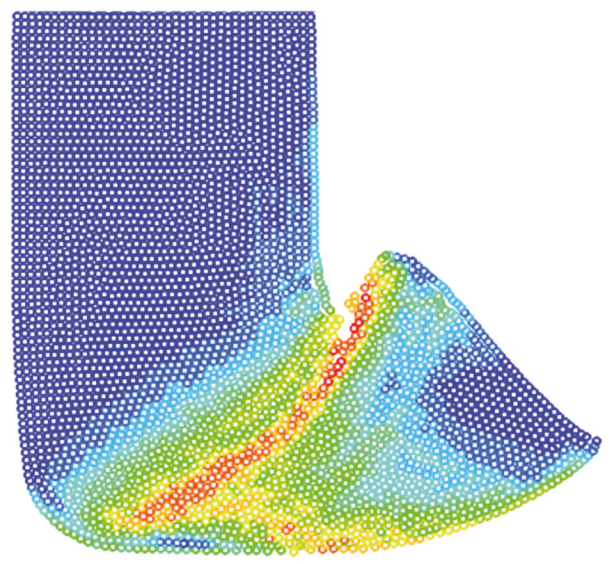

(d)

FIGURE 7: Simulation results of equivalent strain distribution.

strain value of the particle near the shear plane, the specimen initiates cracks at the internal corner of the die.

According to the computation results based on SPH, as shown in Figures 8(a) and 8(b), lamellar cracks appear on the surface of the extruded specimen and extend parallel to the shear plane. The generation of cracks can release the strain concentration within the vicinity, to reduce the damage value accumulation of the surrounding particles and stop the extension of the cracks. Along with the extrusion continuation, the damage evolves repeatedly on the surface of the specimen, resulting in lamellar cracks. A layered distribution in parallel with the crack extension is observed within the high strain concentration area on the extruded specimen.

On viewing the test results provided in Figure 8(c), it can be seen that the results from the program provided herein are consistent with the test results, which demonstrates that the program provided herein, based on $\mathrm{SPH}$, is feasible and valid for conducting damage prediction and determining dynamic crack initiation and extension of ECAP process. of Pure Magnesium with Can. Figure 9 shows the principal diagram of ECAP technology with can. The internal structure of the dies is the same as the traditional ECAP technology. During the canned ECAP process of pure magnesium, the deformation characteristics of the canned material directly influence the plastic deformation of the specimen inside. Therefore, we carry out a simulation study on the deformation process during the ECAP process of pure magnesium canned with two types of materials at room temperature.

The pure magnesium specimen with dimensions of $\Phi 8 \mathrm{~mm} \times 49 \mathrm{~mm}$ before extrusion deformation uses a can with external dimensions of $10 \mathrm{~mm} \times 10 \mathrm{~mm} \times 50 \mathrm{~mm}$, with the size of the inner hole being the same as that of the specimen. In order to facilitate the computation, assume that the contact between any two parts of the specimen, the can and die, is smooth without friction, and the extrusion velocity is $4 \mathrm{~mm} \cdot \mathrm{min}^{-1}$.

Figure 10 shows the initial state of the simulation of ECAP with can by using the SPH method. Before the simulation, we discretely discompose the specimen to be deformed and the canned material into two types of particles, wherein the 6,272 particles in the red area represent the specimen; the 1,728 particles in the purple area represent the canned material; and the particles in the black area denote the boundary. 2024 aluminum alloy and industrial pure iron are adopted as the 


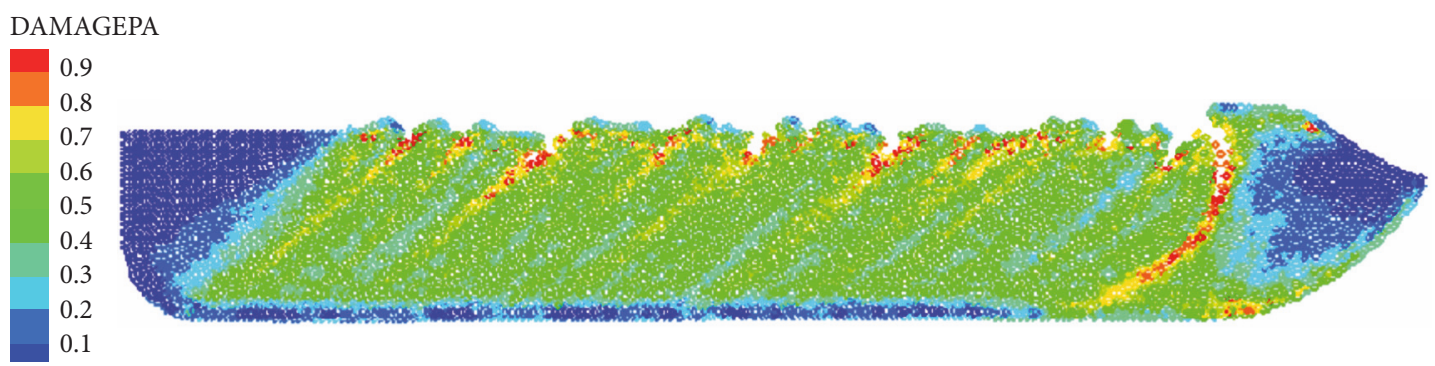

(a)

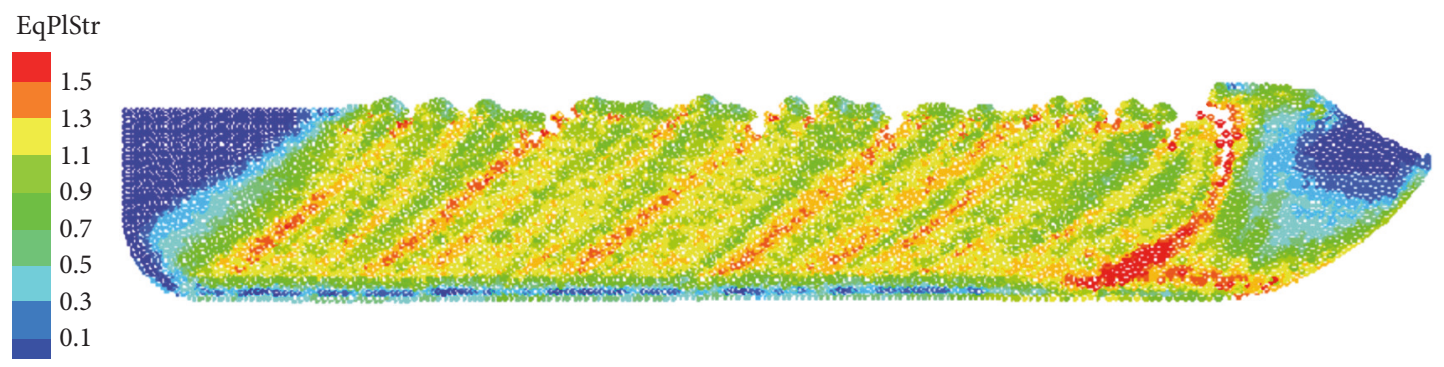

(b)

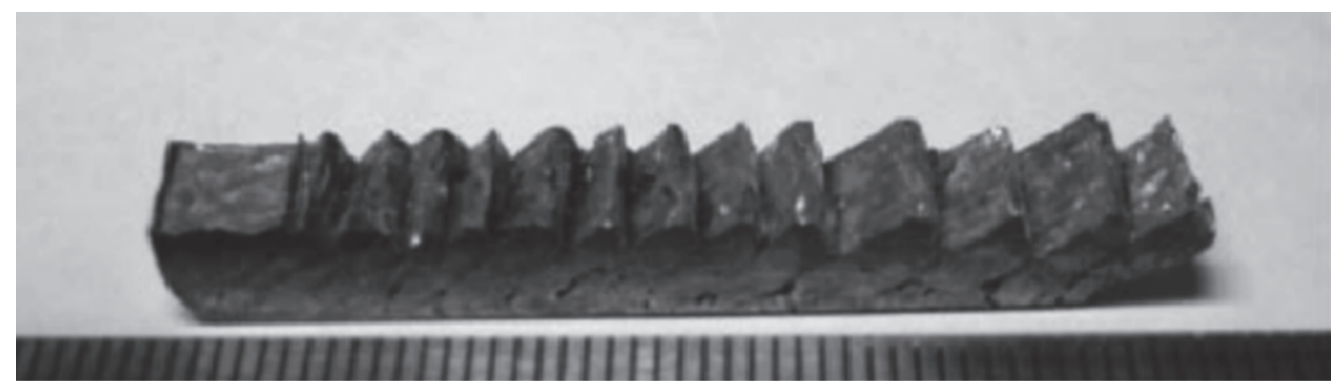

(c)

FIGURE 8: Simulation results of room temperature ECAP of pure magnesium. (a) Computation results of accumulated damage value based on SPH. (b) Computation Results of equivalent strain based on SPH. (c) Test results.

canned material, with the parameters for their constitutive equation listed in Table 2 [29].

Commercial DEFORM-V10.2 software is used for the finite element modeling. The upper die and bottom die are assumed to be discretely rigid, meaning that there is no deformation during the process, which is carried out at room temperature. Also, the heat generation is ignored during the operation. The extrusion velocity is $4 \mathrm{~mm} \cdot \mathrm{min}^{-1}$. The pure magnesium specimen with dimension of $\Phi 8 \mathrm{~mm} \times 49 \mathrm{~mm}$ before extrusion deformation uses a can with external dimensions of $10 \mathrm{~mm} \times 10 \mathrm{~mm} \times 50 \mathrm{~mm}$, with the size of the inner hole being the same as that of the specimen. Compared with the simulation using the finite element method, with the initial state of ECAP with can as shown in Figure 11, the can participates in the deformation during the extrusion. The canned material is set as the plastic model, and the specimen and can are divided into meshes respectively, with 32,000 specimen meshes (yellow area) and 32,000 can meshes (blue area). Adaptive meshing and automatic remeshing are applied and justified for all simulations to prevent mesh failure during large deformations.
3.2.1. Numerical Simulation of Room Temperature ECAP Process of Pure Magnesium Canned with 2024 Aluminum Alloy. The SPH method and finite element method are adopted to simulate the room temperature ECAP deformation process of pure magnesium canned with 2024 aluminum alloy. The results of equivalent strain distribution simulation are shown in Figure 12.

Figure 12(a) shows the results computed using the $\mathrm{SPH}$ method; Figures 12(b) and 12(c), respectively, show the equivalent strain distributions of the can and inside specimen obtained from the simulation based on the finite element method. It can be seen in Figure 12(a) that, during the ECAP process of the combined specimen canned with 2024 aluminum alloy, the can fails to protect the inside specimen, and the deformation of the combined specimen results in severe cracks. The equivalent strain distribution of the inside specimen changes drastically, and a high strain concentration area forms a lamella shape parallel to the shear plane, resulting in the cracks extending within the high strain area. The equivalent strain distribution on the upper surface of the can is not homogeneous, with intermittent high strain areas. 
TABLE 2: Parameters for constitutive equation of canned materials.

\begin{tabular}{lccccccc}
\hline Material & $\rho\left(\mathrm{kg} \cdot \mathrm{m}^{-3}\right)$ & $\nu$ & $A(\mathrm{MPa})$ & $B(\mathrm{MPa})$ & $\mathrm{C}$ & $\mathrm{m}$ \\
\hline industrial pure iron & 7830 & 0.33 & 440 & 510 & 0.014 & 1.03 & 0.26 \\
\hline$T_{r}(\mathrm{~K})$ & $T_{m}(\mathrm{~K})$ & $C_{v}$ & $D_{1}$ & $D_{2}$ & $D_{3}$ & $D_{4}$ \\
\hline 293 & 1793 & 477 & -2.20 & 5.43 & -0.47 & 0.016 \\
\hline Material & $\rho\left(\mathrm{kg} \cdot \mathrm{m}^{-3}\right)$ & $v$ & $A(\mathrm{MPa})$ & $B(\mathrm{MPa})$ & $\mathrm{C}$ & 0.0 \\
\hline 2024 aluminum alloy & 2770 & 0.3 & 345 & 462 & 0.001 & $\mathrm{~m}$ \\
\hline$T_{r}(\mathrm{~K})$ & $T_{m}(\mathrm{~K})$ & $C_{v}$ & $D_{1}$ & $D_{2}$ & $D_{3}$ & 2.75 & $D_{4}$ \\
\hline 293 & 775 & 887 & 0.012 & 0.13 & 1.5 & -0.0175 \\
\hline
\end{tabular}

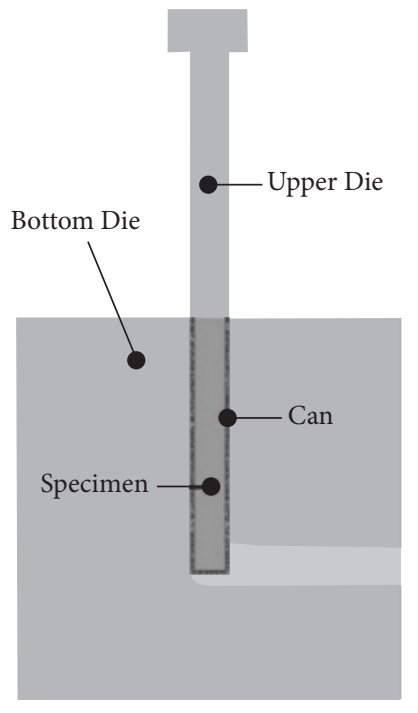

FIgURE 9: Structural diagram of ECAP with can.

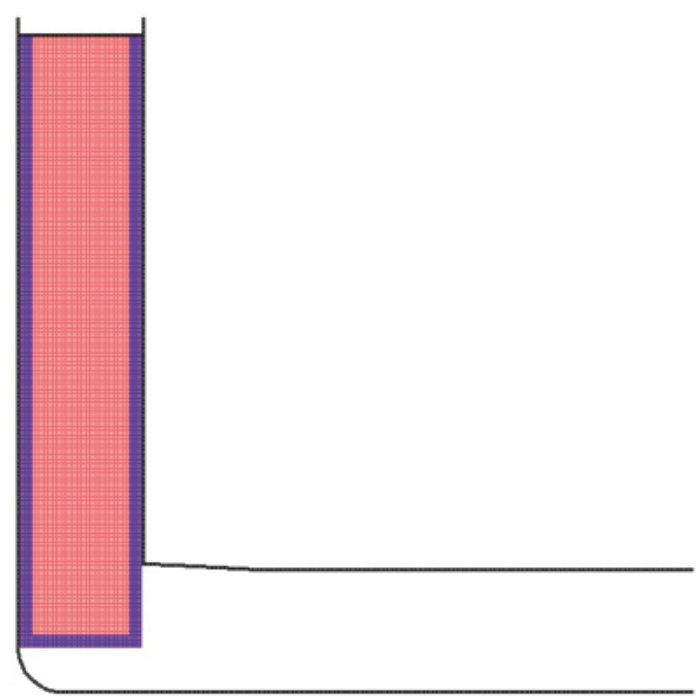

FIGURE 10: ECAP model with can based on SPH.

It can be seen from the results of the finite element simulation in Figure 12(b) that the intermittent high strain concentration areas on the upper surface of the can show a distribution law similar to the results of simulation based on

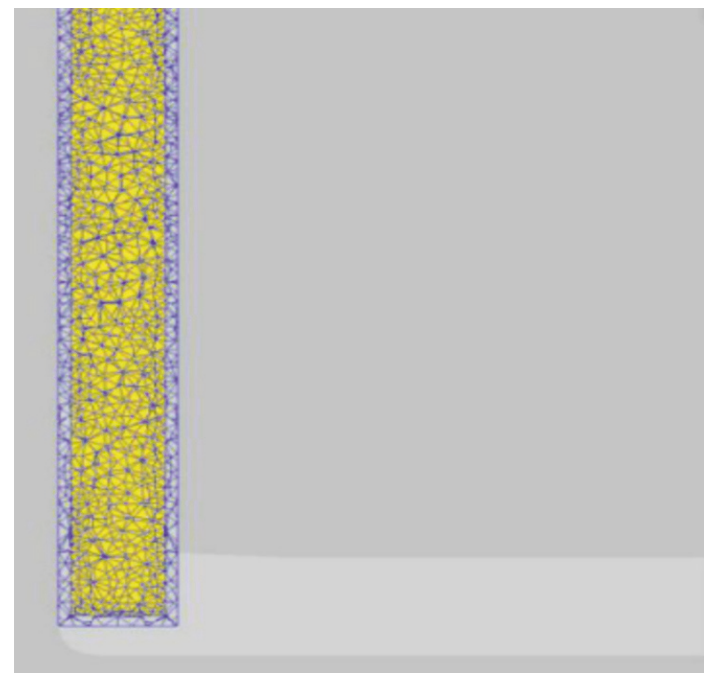

FIGURE 11: ECAP model with can based on finite element method.

the SPH method. It can be further observed in Figure 12(c) that the equivalent strain distribution of the inside specimen shows a law basically identical to the results obtained by using the SPH method. The high strain areas along the extrusion direction are concentrated in a lamellar pattern, and the inside specimen has rough and uneven edges due to the inhomogeneous deformation. Based on the comparison of the results, the equivalent strain distribution law obtained from the program described herein is basically consistent with the results from the finite element software simulation.

Figure 13(a) shows the results of the damage values computed based the SPH program, while Figures 13(b) and 13(c), respectively, show the damage value distribution of the can and inside specimen computed by using the finite element software, and Figure 13(d) shows the test results. According to the results computed by the SPH method, as shown in Figure 13(a), high damage values are concentrated on the crack surfaces and high strain areas and decrease progressively along the shear plane towards the bottom till finally disappearing. Compared with the results of simulation by using the finite element method, as shown in Figures 13(b) and $13(\mathrm{c})$, large damage value areas appear on the upper surface of the can and show a distribution law similar to the results obtained by using the SPH method.

Our research group also conducted a test on the ECAP process of pure magnesium canned with 2024 aluminum 


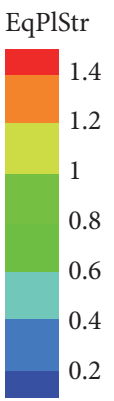

2.67
2.40
2.14
1.87
1.60
1.34
1.07
0.801
0.534
0.267
0.100

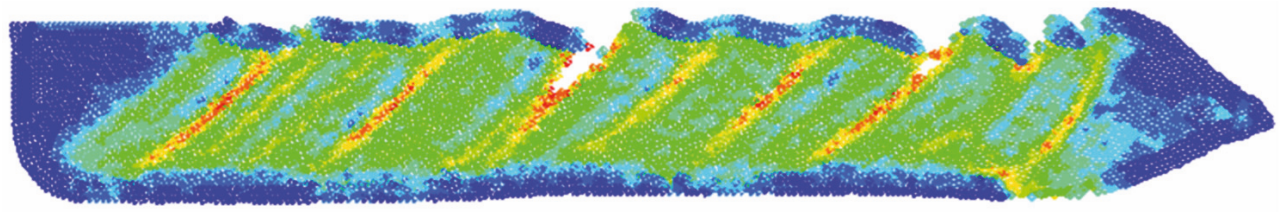

(a)

EqPIStr



(b)

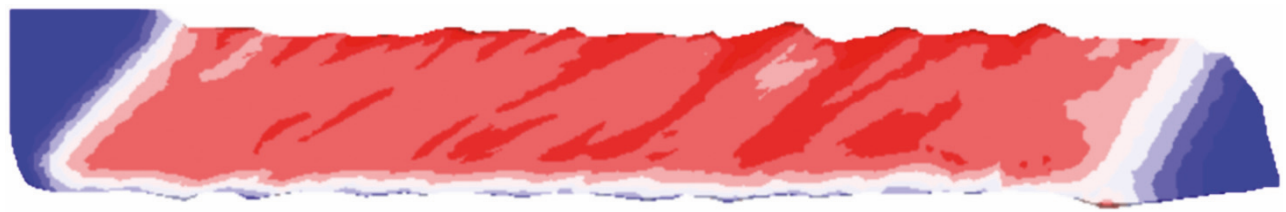

(c)

FiguRE 12: Simulation results of equivalent strain distribution. (a) Computation results based on SPH. (b), (c) Computation results based on finite element method.

alloy, with the macrostructure of extruded combined specimen shown in Figure 13(d). It can be seen, by comparison, that the results computed based on the SPH program are basically consistent with the test results.
3.2.2. Numerical Simulation of Room Temperature ECAP Process of Pure Magnesium Canned with Industrial Pure Iron. The SPH program and the finite element software were used to, respectively, simulate and study the room temperature 
DAMAGEPA

0.9
0.8
0.7
0.6
0.5
0.4
0.3
0.2
0.1

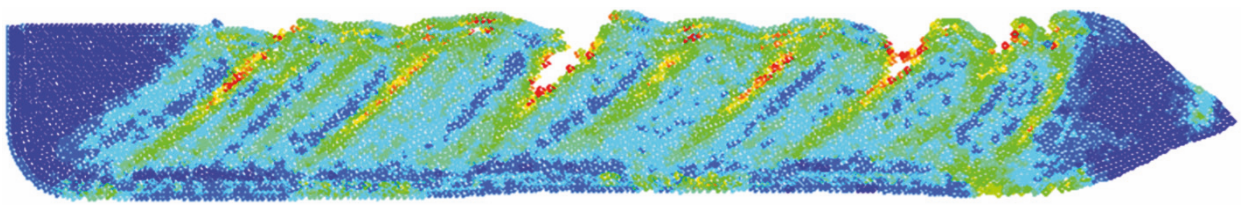

(a)
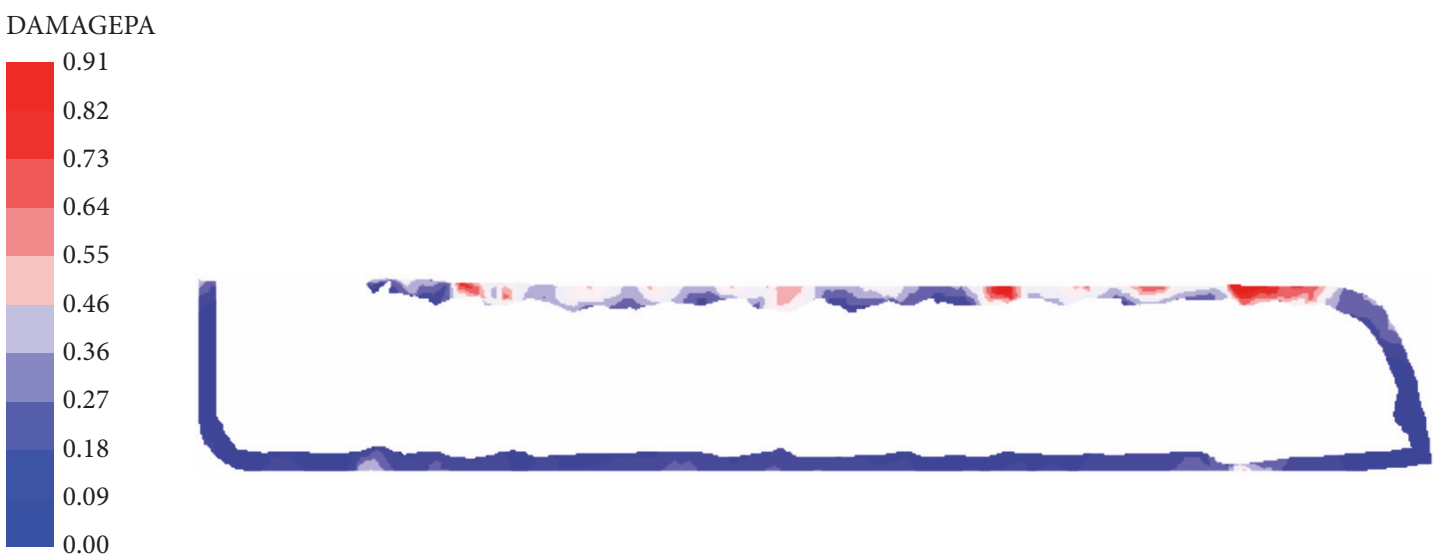

(b)

DAMAGEPA
0.30
0.27
0.24
0.21
0.18
0.15
0.12
0.09
0.06
0.03
0.00

(c)

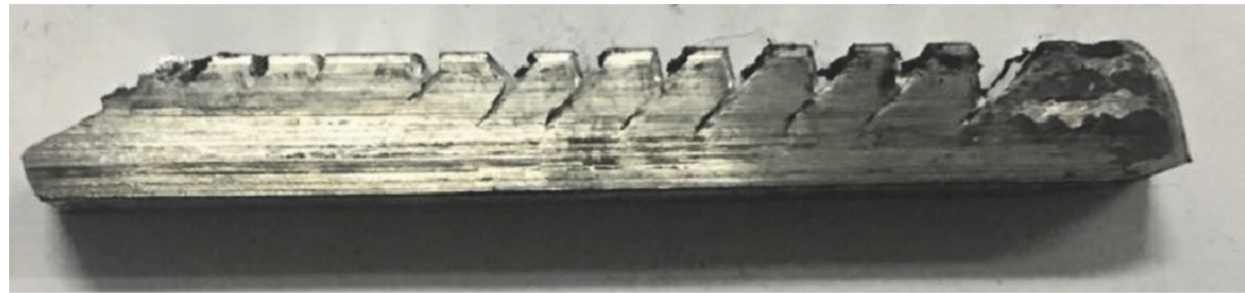

(d)

FIGURE 13: Simulated results of damage value distribution and test results. (a) Computation results based on SPH. (b), (c) Simulation results based on finite element method. (d) Test results. 
ECAP deformation process of pure magnesium material canned with industrial pure iron. The computation results of the equivalent strain distributions are shown in Figure 14.

As seen from Figure 14, the pure iron material provides sufficient triaxial compressive stresses to successively conduct the room temperature ECAP deformation process of pure magnesium material. Figure 14(a) shows the results of the equivalent strain distribution computed by using the $\mathrm{SPH}$ method, while Figures 14(b) and 14(c) show the results of the equivalent strain distributions of the can and the inside specimen simulated by using the finite element method. It can be observed from the equivalent strain distribution of the inside specimen, as shown in Figure 14(a), that the plastic deformation of pure magnesium is very homogeneous, whether it is generated in the vertical direction or in the extrusion direction. As shown in Figure 14(b), high strain concentration occurs on the head of the can close to the upper surface, but differs from the results based on the SPH computation. The equivalent strain distribution of the inside specimen, as shown in Figure 14(c), shows a law similar to the results obtained by using the SPH method. The ECAP deformation process of pure magnesium canned with industrial pure iron is further analyzed below from the perspective of accumulated damage value.

Figure 15(a) shows the simulation results of the damage values based the SPH program, while Figures 15(b) and 15(c), respectively, show the combined specimen damage value distributions in the simulation results obtained by using the finite element software. Figure 15(d) shows the macrostructure test results. It can be observed from Figures 15(a) and 15(c), the damage value distributions of the inside specimen by using the SPH method and finite element method, respectively, that the maximum damage values of the inside specimen obtained by the SPH method and the finite element method are both less than 0.16 , with both values being far lower than the critical fracture accumulated damage value. It can be seen in Figures 15(a) and 15(d) that the results obtained from the SPH simulation are similar to those from the testing.

To better observe the changes of strain values and damage values in the ECAP process, 9 points (P1-P9) are arranged on the major deformation area of the inside specimen along the extrusion direction (ED) as shown in Figure 16.

Figure $17(a)$ is the equivalent strain curves of specimen canned with industrial pure iron, and Figure $17(\mathrm{~b})$ is the equivalent strain curves of specimen canned with 2024 aluminum alloy. According to Figure 17(a), the equivalent strain values of the inside specimen obtained by the SPH method and the finite element method are similar in trend, and the equivalent strain values at each point located in the major deformation area have small difference. The average equivalent strain value of the specimen canned with industrial pure iron in the major deformation area is 1.31 obtained by using the SPH method, which is similar to the finite element simulation result in which the average equivalent strain value of the major deformation area is 1.24. From Figure 17(b), the equivalent strain values have large fluctuation. According to the SPH computation results, the average equivalent strain value of the specimen canned with 2024 aluminum alloy is
1.19 within the major deformation area. It can be seen from the results of the finite element simulation that the average equivalent strain value of the specimen canned with 2024 aluminum alloy is 1.13 .

There are two damage value curves obtained by using the SPH method in Figure 18. When adopting 2024 aluminum alloy as the canned material, the damage values of specimen are large. When adopting industrial pure iron as the canned material, the damage values of specimen are relatively small. From Figure 18, we can see that the types of external can materials will directly influence the plastic deformation of internal specimen.

From the above, when the head of the combined specimen enters into the plastic deformation area, the can needs to provide sufficient triaxial compressive stresses on the inside specimen, so as to avoid the specimen cracking due to the high strain concentration, and to ensure the deformation homogeneity of the inside specimen. By comparing the results of the simulations using 2024 aluminum alloy and industrial pure iron as canned materials, it is evident that the 2024 aluminum alloy cannot provide sufficient triaxial compressive stresses, and the canned material on the upper surface of combined specimen is broken along with the cracking of inside specimen, while the industrial pure iron can not only provide favorable protection for the pure magnesium, but also improve the deformation homogeneity of pure magnesium.

\section{Conclusions}

Based on SPH, a mathematical model is established for numerical simulation of the ECAP processing of can under room temperature conditions. The simulation results based on the SPH method are compared with the simulation results based on the finite element method. In order to further verify the simulation results based on SPH, some controlled tests were conducted. In addition, an ECAP die was designed. The crossing angle was $90^{\circ}$, and there was a $3^{\circ}$ deflection angle structure above the horizontal channel.

(1) In this study, based on the SPH method, we utilize the invalid particles and crack treatment techniques, building an ECAP mathematical model incorporating damage prediction, in order to simulate crack initiation and dynamic extension in the ECAP process. By taking pure magnesium as an example, a numerical simulation of the room temperature ECAP process without can was conducted to verify the feasibility and validity of the mathematical model. A numerical simulation of the room temperature ECAP process with can was conducted to demonstrate that the types of external can materials directly influence the plastic deformation of internal specimen.

(2) When simulation of pure magnesium during ECAP at room temperature is conducted using industrial pure iron as the canned material, the simulation results based on SPH show that the plastic deformation of the pure magnesium specimen is homogeneous in both the vertical direction and the extrusion direction. The average equivalent strain value of the specimen in the major deformation area is 1.31 , which is similar to the finite element simulation result with the average 


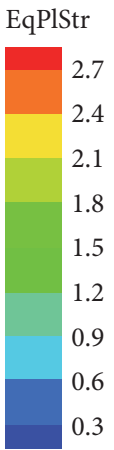

EqPIStr

2.56
2.30
2.05
1.79
1.54
1.28
1.02
0.768
0.512
0.256
0.000

EqPIStr

1.59
1.43
1.27
1.11
0.954
0.795
0.636
0.477
0.318
0.159
0.000

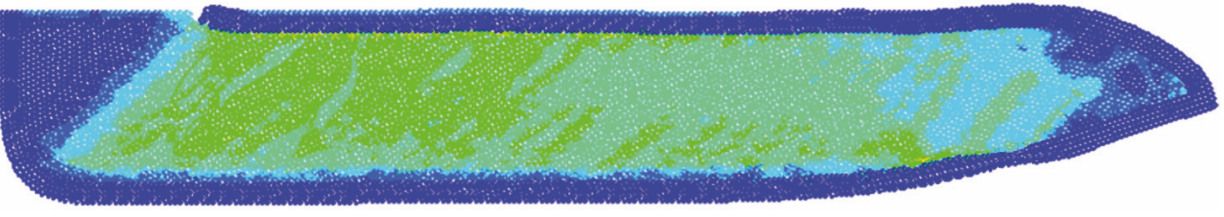

(a)

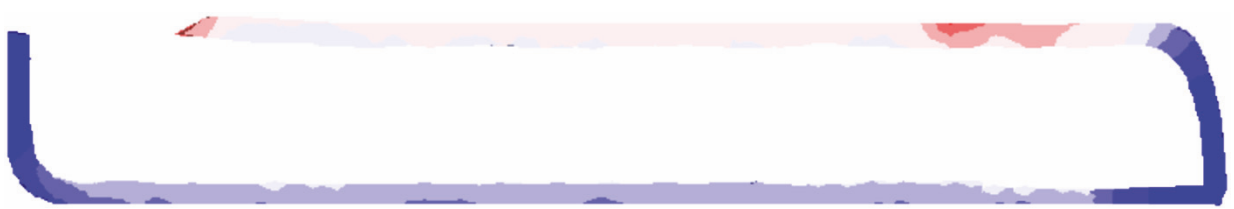

(b)

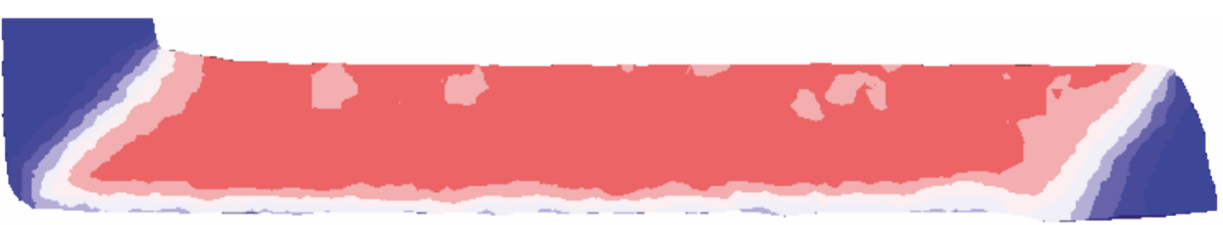

(c)

FIGURE 14: Computation results of equivalent strain distribution. (a) Computation results based on SPH. (b), (c) Computation results based on finite element method. 
DAMAGEPA
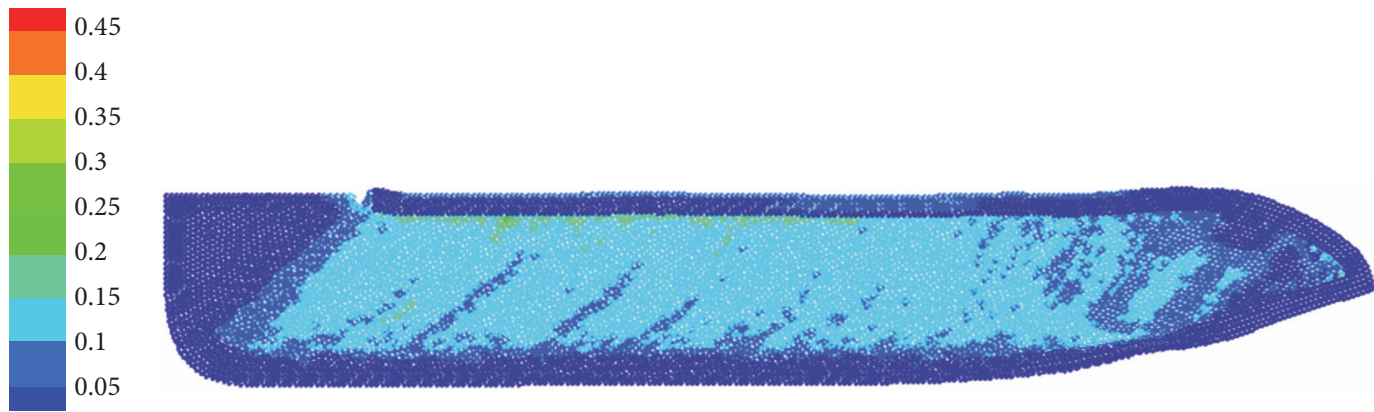

(a)

DAMAGEPA

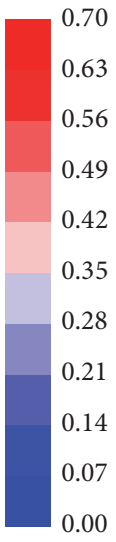

(b)

DAMAGEPA
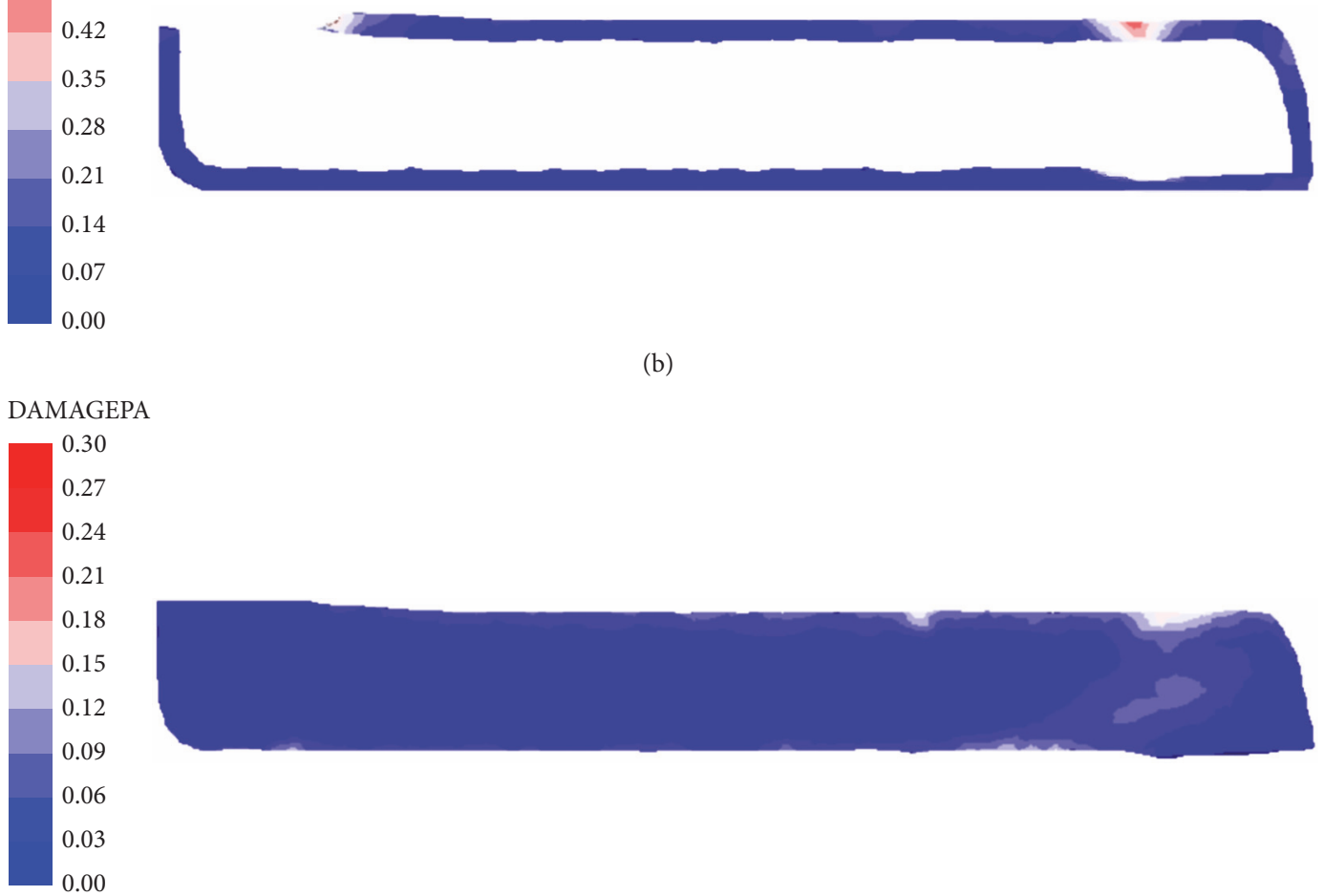

(c)

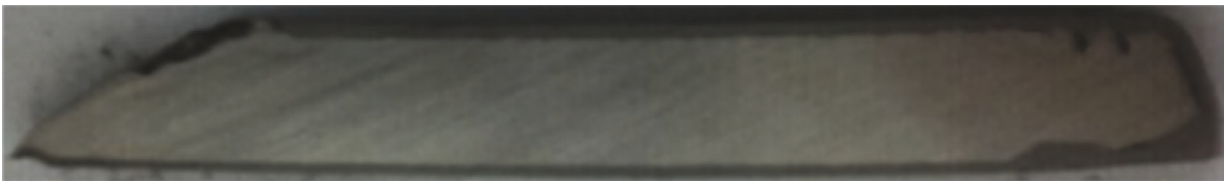

(d)

FIGURE 15: Simulated results of damage value distribution and test results. (a) Computation results based on SPH. (b), (c) Simulation results based on finite element method. (d) Test results.

equivalent strain value of the major deformation area of 1.24 . From the damage perspective, the maximum damage values of the inside specimen obtained by the SPH method and the finite element method are both less than 0.16 , with both values being far lower than the critical fracture accumulated damage value. After analysis, the reason is that when the combined specimen enters the plastic deformation area, the industrial pure iron can is able to provide sufficient triaxial 


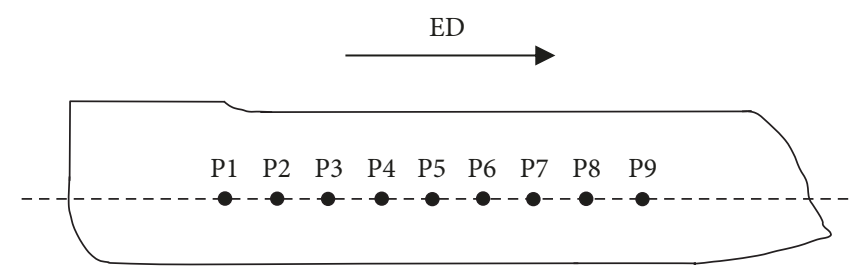

FIGURE 16: Distribution of observation points.



(a)

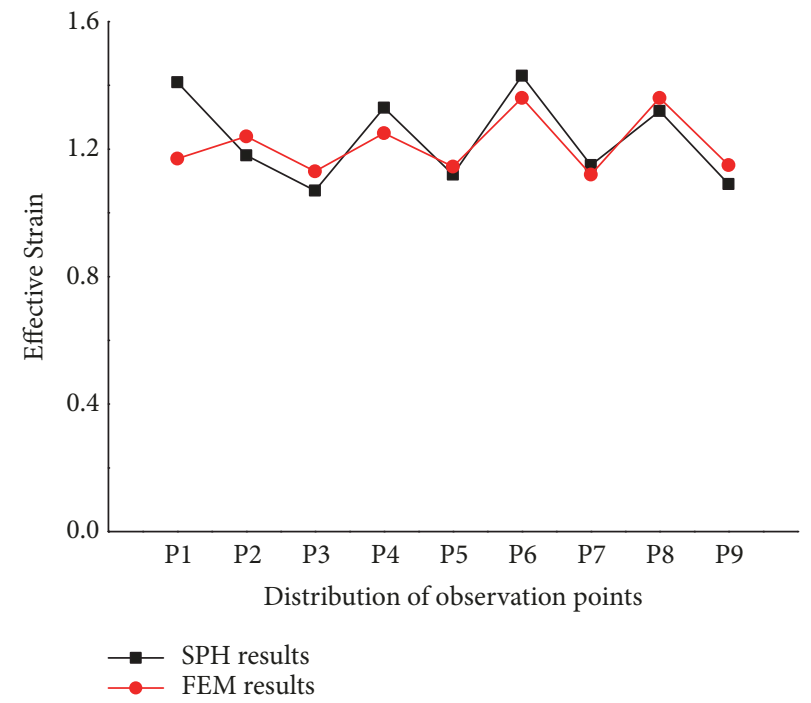

(b)

FIGURE 17: Comparison of equivalent strain curves. (a) Specimen canned with industrial pure iron. (b) Specimen canned with 2024 aluminum alloy.



FIGURE 18: Comparison of damage value curves obtained by using the SPH method.

compressive stresses on the inside specimen, avoiding the specimen cracks due to the high strain concentration, and to ensure the deformation homogeneity of the inside specimen.
When simulation of pure magnesium during ECAP at room temperature is conducted using 2024 aluminum alloy as the canned material, the canned material on the upper surface of specimen is broken along with cracking of the inside specimen. The test results agree with the above simulation results, further explaining the correctness of the mathematical model established in this paper.

\section{Data Availability}

The data used to support the findings of this study are available from the corresponding author upon request.

\section{Conflicts of Interest}

The authors declare that there are no conflicts of interest regarding the publication of this article.

\section{Acknowledgments}

This research has been financially supported by the National Natural Science Foundation of China (NO. 51874209, 51574176) and Major Research \& Development Plan of Shanxi Province (International Cooperation project) (201603D421028). 


\section{References}

[1] B. Li, B. Teng, and G. Chen, "Microstructure evolution and mechanical properties of Mg-Gd-Y-Zn-Zr alloy during equal channel angular pressing," Materials Science and Engineering, vol. 744, pp. 396-405, 2019.

[2] T. Krajňák, P. Minárik, J. Stráský, K. Máthis, and M. Janeček, "Mechanical properties of ultrafine-grained AX41 magnesium alloy at room and elevated temperatures," Materials Science and Engineering, vol. 731, pp. 438-445, 2018.

[3] W. Lei, W. Liang, H. Wang, and H. Guo, "Evolution of texture and mechanical properties of pure mg processed by ECAP at room temperature," JOM: The Journal of The Minerals, Metals \& Materials Society (TMS), vol. 69, no. 11, pp. 2297-2301, 2017.

[4] S. Biswas, S. Singh Dhinwal, and S. Suwas, "Room-temperature equal channel angular extrusion of pure magnesium," Acta Materialia, vol. 58, no. 9, pp. 3247-3261, 2010.

[5] F. Djavanroodi, M. Daneshtalab, and M. Ebrahimi, "A novel technique to increase strain distribution homogeneity for ECAPed materials," Materials Science and Engineering, vol. 535, pp. 115-121, 2012.

[6] H. Zhijun, Z. Dai, and W. Liang, "Microstructure and mechanical property of pure mg deformed by canned equal channel angular pressing at room temperature," Hot Working Technology, vol. 46, no. 11, pp. 1-4, 2017.

[7] S. C. Yoon, H.-G. Jeong, S. Lee, and H. S. Kim, "Analysis of plastic deformation behavior during back pressure equal channel angular pressing by the finite element method," Computational Materials Science, vol. 77, pp. 202-207, 2013.

[8] F. Djavanroodi and M. Ebrahimi, "Effect of die channel angle, friction and back pressure in the equal channel angular pressing using 3D finite element simulation," Materials Science and Engineering, vol. 527, no. 4-5, pp. 1230-1235, 2010.

[9] M. Ebrahimi, S. Attarilar, C. Gode, and F. Djavanroodi, "Damage prediction of 7025 aluminum alloy during equal-channel angular pressing," International Journal of Minerals, Metallurgy and Materials, vol. 21, no. 10, pp. 990-998, 2014.

[10] J. Kwon, "Smoothed particle hydrodynamics model for simulating miscible multi-fluid flow," Journal of Computational Physics, vol. 384, pp. 114-133, 2019.

[11] G. G. Pereira, P. W. Cleary, and V. Lemiale, "SPH method applied to compression of solid materials for a variety of loading conditions," Applied Mathematical Modelling, vol. 44, pp. 72-90, 2017.

[12] Y. Lin, G. R. Liu, and G. Wang, "A particle-based free surface detection method and its application to the surface tension effects simulation in smoothed particle hydrodynamics (SPH)," Journal of Computational Physics, vol. 383, pp. 196-206, 2019.

[13] K. Ng, Y. Ng, T. Sheu, and A. Mukhtar, "Fluid-solid conjugate heat transfer modelling using weakly compressible smoothed particle hydrodynamics," International Journal of Mechanical Sciences, vol. 151, pp. 772-784, 2019.

[14] Y. Wu, J. Lu, S. Tan, F. Jiang, and J. Sun, "Modified implementation strategy in explosive welding for joining between precipitate-hardened alloys," Journal of Manufacturing Processes, vol. 36, pp. 417-425, 2018.

[15] W. Pan, D. Li, A. M. Tartakovsky, S. Ahzi, M. Khraisheh, and M. Khaleel, "A new smoothed particle hydrodynamics nonnewtonian model for friction stir welding: process modeling and simulation of microstructure evolution in a magnesium alloy," International Journal of Plasticity, vol. 48, pp. 189-204, 2013.
[16] P. W. Cleary, M. Prakash, R. Das, and J. Ha, "Modelling of metal forging using SPH," Applied Mathematical Modelling, vol. 36, no. 8, pp. 3836-3855, 2012.

[17] A. J. C. Crespo, J. M. Domínguez, B. D. Rogers et al., "DualSPHysics: Open-source parallel CFD solver based on Smoothed Particle Hydrodynamics (SPH)," Computer Physics Communications, vol. 187, pp. 204-216, 2015.

[18] Q. Hongfu, New Method and Application of Smooth Particle Hydrodynamics, China Science Publishing \& Media Ltd., Beijing, China, 2017.

[19] G. R. Liu and M. B. Liu, Smoothed Particle Hydrodynamics: A Mesh-Free Particle Method, World Scientific Publishing Co. Pte. Ltd., Singapore, 2003.

[20] T. Børvik, M. Langseth, O. S. Hopperstad, and K. A. Malo, "Ballistic penetration of steel plates," International Journal of Impact Engineering, vol. 22, no. 9-10, pp. 855-886, 1999.

[21] M. M. Rahaman, A. Pathak, and D. Roy, "A thermo-viscoplastic damage model and SPH simulations of plugging failure," Mechanics of Advanced Materials and Structures, vol. 25, pp. 1374-1382, 2017.

[22] J. J. Monaghan, "SPH without a tensile instability," Journal of Computational Physics, vol. 159, no. 2, pp. 290-311, 2000.

[23] T. Ye, D. Pan, C. Huang, and M. Liu, "Smoothed particle hydrodynamics (SPH) for complex fluid flows: Recent developments in methodology and applications," Physics of Fluids, vol. 31, no. 1, Article ID 011301, 2019.

[24] J. J. Monaghan and J. B. Kajtar, "SPH particle boundary forces for arbitrary boundaries," Computer Physics Communications, vol. 180, no. 10, pp. 1811-1820, 2009.

[25] L. Weiwei, Microstructure and Mechanical Properties of Pure $\mathrm{Mg}$ After ECAP at Room Temperature, Taiyuan University of Technology, Taiyuan, China, 2016.

[26] Z. Huang, Deformation Mechanism and Microstructure and Property Evolution of Pure $\mathrm{Mg}$ during Canned ECAP at Room Temperature, Taiyuan University of Technology, Taiyuan, China, 2017.

[27] W. Zhang, X.-K. Xiao, and G. Wei, "Constitutive relation and fracture model of 7A04 aluminum alloy," Combustion, Explosion, and Shock Waves, vol. 31, no. 1, pp. 81-87, 2011.

[28] W. Zhang, G. Wei, and X.-K. Xiao, "Constitutive relation and fracture criterion of $2 \mathrm{~A} 12$ aluminum alloy," Acta Armamentarii, vol. 34, no. 3, pp. 276-282, 2013.

[29] F. Sicong and Q. Sun, "Investigation on parameters calibration for the J-C failure model of aluminum alloy," Computer Simulation, vol. 30, no. 9, pp. 46-50, 2013. 


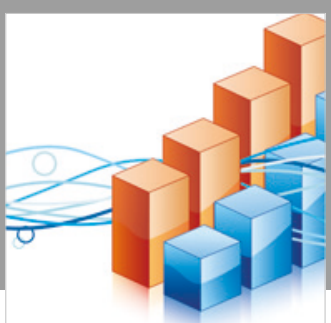

Advances in

Operations Research

\section{-n-m}
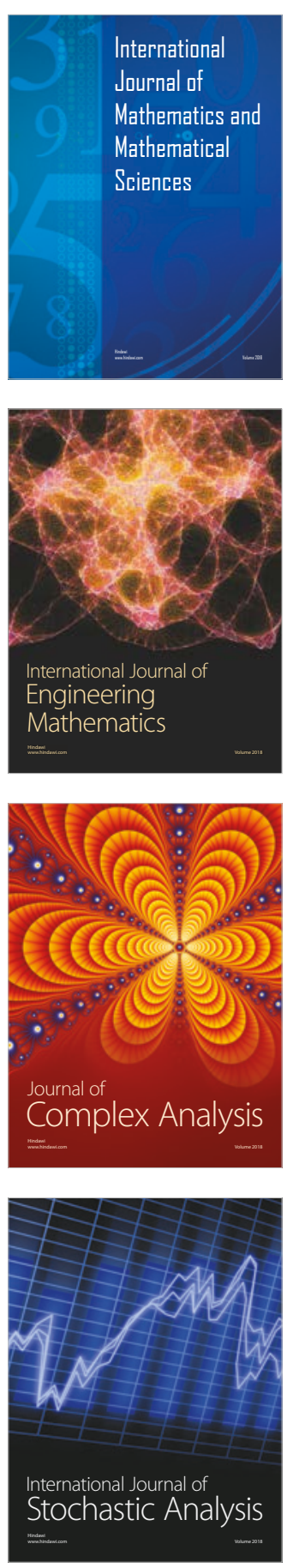
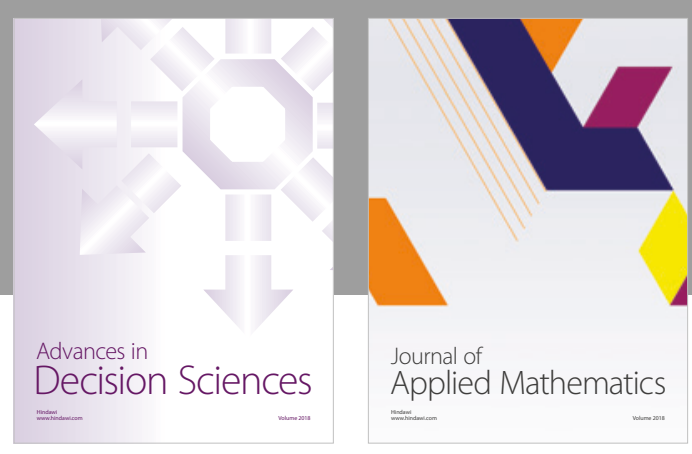

Journal of

Applied Mathematics
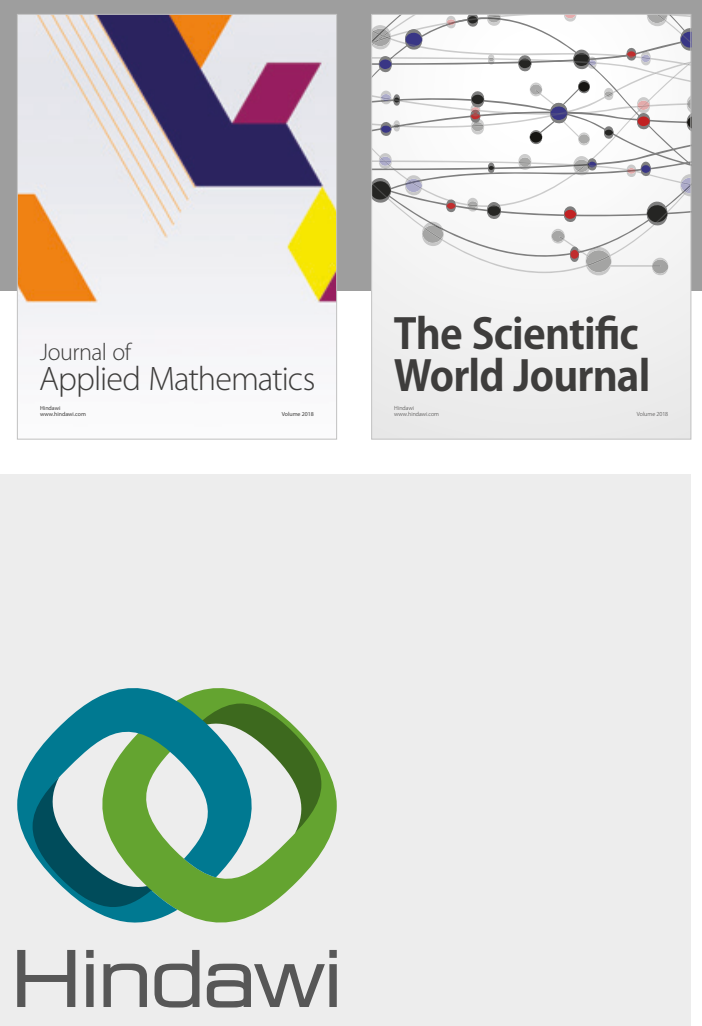

Submit your manuscripts at

www.hindawi.com

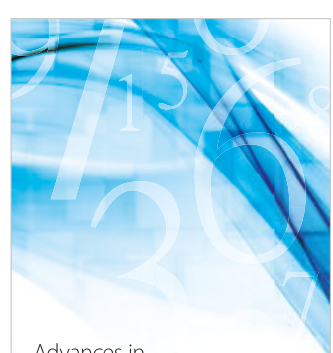

Advances in
Numerical Analysis
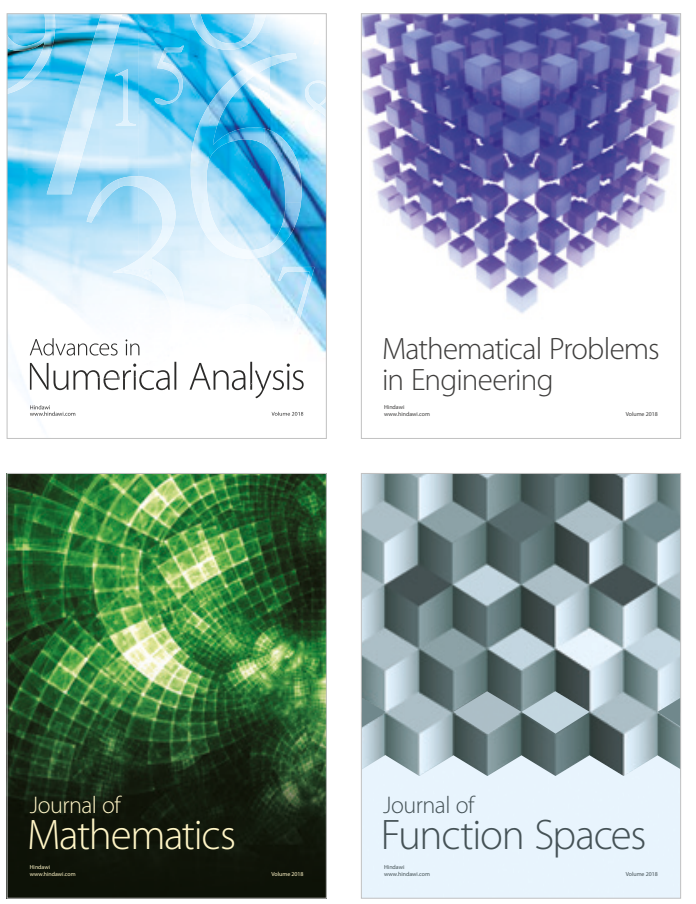

Mathematical Problems in Engineering

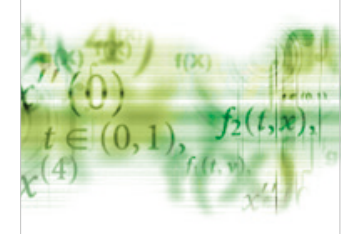

International Journal of

Differential Equations



Journal of

Function Spaces
The Scientific

World Journal



Journal of

Probability and Statistics
